\title{
A NOTE ON ERGODIC TRANSFORMATIONS
}

\author{
J. R. BLUM ${ }^{1}$ AND D. L. HANSON ${ }^{2}$
}

1. Let $(\Omega, Q, P)$ be a probability space and let $T$ be a 1-1 point transformation mapping $\Omega$ onto $\Omega$ which is bimeasurable and measure preserving with respect to $P$. $T$ is ergodic with respect to $(\Omega, Q, P)$ provided $P(A)=0$ or 1 for every $A \in Q$ which is invariant with respect to $T$. It is well known that

(i) $T$ is ergodic if and only if

(ii) $\lim _{n \rightarrow \infty} \sum_{i=0}^{n-1} P\left(A T^{i} B\right) / n=P(A) P(B)$ for every $A \in Q, B \in Q$. That (ii) implies (i) is obvious. It follows as a consequence of the mean ergodic theorem that (i) implies (ii) uniformly in either variable set for each fixed value of the other variable set. (A counterexample can be given to simultaneous uniformity in both variable sets.)

In $\$ 2$ we prove a convergence theorem for set functions using the properties of uniform absolute continuity and a type of asymptotic invariance. In $\S 3$ we use this theorem to show that (i) implies

(iii) $\lim _{n \rightarrow \infty}\left(1 / n^{N}\right) \cdot \sum_{i_{1}=0}^{n-1} \cdots \sum_{i_{N}=0}^{n-1} P\left(A_{0} T^{i_{1}} A_{1} \cdots T^{i_{N}} A_{N}\right)=\prod_{i=0}^{N} P\left(A_{i}\right)$

for each choice of $N$ and that if $N$ of the sets $A_{0}, \cdots, A_{N}$ are fixed then the convergence is uniform in the other set. The above result can be obtained rather easily from the mean ergodic theorem, however, using the mean ergodic theorem the authors have been able to obtain uniformity only in $A_{0}$. The method of proof using the theorem of $\$ 2$ gives uniformity in an arbitrary $A_{i}$ and shows that averaging is used only to guarantee uniform absolute continuity and asymptotic invariance.

2. We shall need the following result, proved in [1]:

Lemma. Let $(\Omega, Q, P)$ be a probability space and let $T$ be ergodic with respect to $(\Omega, Q, P)$. Let $\left\{Q_{n}, n \geqq 1\right\}$ be a sequence of probability measures defined on $a$ and satisfying

(i) $\lim _{n \rightarrow \infty}\left|Q_{n}(A)-Q_{n}(T A)\right|=0$ for every $A \in Q$, and

(ii) the measures $Q_{n}$ are uniformly absolutely continuous with respect to $P$. Then $\lim _{n \rightarrow \infty} Q_{n}(A)=P(A)$ for every $A \in Q$.

Received by the editors April 2, 1962 and, in revised form, June 28, 1962.

1 Research supported by the National Science Foundation, Grant NSF-G21219.

2 Research supported by the Atomic Energy Commission. 
TheOREM. Assume

(i) $(\Omega, a, P)$ is a probability space and $T$ is ergodic with respect to $(\Omega, a, P)$.

(ii) $I$ is an index set and for each positive integer $n, I_{n}$ is a nonempty subset of $I$.

(iii) $N$ is a nonnegative integer and for each $i \in I$ there is a function $Q_{i}\left(A_{0}, \cdots, A_{N}\right)$ defined for every $N+1$ sets $A_{0}, \cdots, A_{N}$ with $A_{i} \in Q$ such that

(a) $Q_{i}(\Omega, \cdots, \Omega) \equiv 1$.

(b) If any $N$ of the arguments of $Q_{i}$ are fixed then $Q_{i}$ is a measure on a with respect to the remaining argument and the family $\left\{Q_{i}\right\}$ of these measures is uniformly absolutely continuous with respect to $P$.

(c) If $A_{0}, \cdots, A_{N}$ are sets with $A_{i} \in Q$ and $k$ is an integer with $0 \leqq k \leqq N$ then

$$
\begin{aligned}
\lim _{n \rightarrow \infty} \sup _{i \in I_{n}} \mid Q_{i}\left(A_{0}, \cdots, A_{N}\right) & \\
& -Q_{i}\left(A_{0}, \cdots, A_{k-1}, T A_{k}, A_{k+1}, \cdots, A_{N}\right) \mid=0 .
\end{aligned}
$$

Then

$$
\lim _{n \rightarrow \infty} \sup _{i \in I_{n}}\left|Q_{i}\left(A_{0}, \cdots, A_{N}\right)-\prod_{i=0}^{N} P\left(A_{i}\right)\right|=0
$$

for every $N+1$ sets $A_{0}, \cdots, A_{N}$ with $A_{i} \in$ a.

Proof. Assume henceforth that all sets under discussion are elements of $a$. We shall proceed by induction on $N$. For $N=0$ the conclusion follows immediately from the lemma. Assume then that $N>0$ and the induction hypothesis for $N-1$. Let $A_{0}, \cdots, A_{N}$ be any sets. The family of functions $\left\{Q_{i}\left(A_{0}, \cdots, A_{N-1}, \Omega\right)\right\}$ satisfies the conditions of the theorem for $N-1$ and the induction hypothesis is that

$$
\lim _{n \rightarrow \infty} \sup _{i \in I_{n}}\left|Q_{i}\left(A_{0}, \cdots, A_{N-1}, \Omega\right)-\prod_{i=0}^{N-1} P\left(A_{i}\right)\right|=0 .
$$

If $P\left(A_{i}\right)=0$ for some $i$ we are done, so assume $\prod_{i=0}^{N-1} P\left(A_{i}\right)>0$. For each $n=1,2, \cdots$ let $k_{n} \in I_{n}$ and choose $n$ so large that $Q_{k_{n}}\left(A_{0}, \cdots, A_{N-1}, \Omega\right)>0$. For $n$ in this range define

$$
P_{n}(A)=\frac{Q_{k_{n}}\left(A_{0}, \cdots, A_{N-1}, A\right)}{Q_{k_{n}}\left(A_{0}, \cdots, A_{N-1}, \Omega\right)} .
$$

Then it is easily verified that the sequence $\left\{P_{n}(A)\right\}$ satisfies the hypotheses of the lemma and consequently $\lim _{n \rightarrow \infty} P_{n}(A)=P(A)$ for every $A$. In particular for $A=A_{N}$ we obtain $\lim _{n \rightarrow \infty} Q_{k_{n}}\left(A_{0}, \cdots, A_{N}\right)$ 
$=\prod_{i=0}^{N} P\left(A_{i}\right)$. Since this is true for every such sequence $\left\{k_{n}\right\}$ the theorem follows.

3. As a consequence of the theorem we obtain at once

CoRollary 1. Let $(\Omega, Q, P)$ be a probability space and suppose $T$ is ergodic with respect to $(\Omega, Q, P)$. Let $N$ be a positive integer. Then

$$
\lim _{n \rightarrow \infty} \sum_{i_{1}=0}^{n-1} \cdots \sum_{i_{N}=0}^{n-1} P\left(A_{0} T^{i_{1}} A_{1} \cdots T^{i_{N}} A_{N}\right) / n^{N}=\prod_{i=0}^{N} P\left(A_{i}\right)
$$

for every $N+1$ sets $A_{0}, \cdots, A_{N}$. If any $N$ of the sets are held fixed the convergence is uniform in the remaining set.

Proof. We shall hold $A_{0}, \cdots, A_{N-1}$ fixed since the proof is the same in any other case. Let $\epsilon>0$ and define $a_{\epsilon}=\{A \in Q \mid P(A) \geqq \epsilon\}$. For $A \in Q_{\epsilon}$ let

$$
\begin{aligned}
Q_{n, A}\left(A_{0}, \cdots, A_{N-1}\right) & \\
& =\sum_{i_{1}=0}^{n-1} \cdots \sum_{i_{N}=0}^{n-1} P\left(A_{0} T^{i_{1}} A_{1} \cdots T^{i_{N-1}} A_{N-1} T^{i_{N}} A\right) /\left[n^{N} P(A)\right] .
\end{aligned}
$$

From the theorem it follows immediately that

$$
\lim _{n \rightarrow \infty} Q_{n, A}\left(A_{0}, \cdots, A_{N-1}\right)=\prod_{i=0}^{N-1} P\left(A_{i}\right)
$$

or equivalently

$$
\begin{gathered}
\lim _{n \rightarrow \infty} \sum_{i_{1}=0}^{n-1} \cdots \sum_{i_{N}=0}^{n-1} P\left(A_{0} T^{i_{1}} A_{1} \cdots T^{i_{N-1}} A_{N-1} T^{i_{N}} A\right) / n^{N} \\
=\prod_{i=0}^{N-1} P\left(A_{i}\right) P(A)
\end{gathered}
$$

uniformly for $A \in a_{e}$. The remainder of the proof is clear.

Corollary 1 can be improved slightly.

CoROllary 2. Let $\left\{A_{n}, n \geqq 0\right\}$ be a sequence of sets and let $\left\{N_{n}, n \geqq 0\right\}$ be a sequence of positive integers with $\lim _{n \rightarrow \infty} N_{n}=\infty$. Then

$$
\lim _{n \rightarrow \infty}\left|\sum_{i_{1}=0}^{n-1} \cdots \sum_{i_{N_{n}}=0}^{n-1} P\left(A_{0} T^{i_{1}} A_{1} \cdots T^{i N_{n}} A_{N_{n}}\right) / n^{N_{n}}-\prod_{i=0}^{N_{n}} P\left(A_{i}\right)\right|=0 .
$$

Furthermore if all of the sets in the sequence with the exception of one are held fixed then the convergence is uniform in the remaining set.

Proof. In the proof we shall assume that $A_{1}, A_{2}, \cdots$ are held 
fixed while $A_{0}$ is allowed to vary. The proof is the same in every other case. There are two possibilities:

(a) Suppose $\lim _{N \rightarrow \infty} \prod_{i=1}^{N} P\left(A_{i}\right)=0$. Choose $\epsilon>0$ and let $N$ be so large that $\prod_{i-1}^{N} P\left(A_{i}\right)<\epsilon / 2$. From Corollary 1 we have that

$$
\lim _{n \rightarrow \infty} \sum_{i_{1}=0}^{n-1} \cdots \sum_{i_{N}=0}^{n-1} P\left(A_{0} T^{i_{1}} A_{1} \cdots T^{i_{N}} A_{N}\right) / n^{N}=\prod_{i=0}^{N} P\left(A_{i}\right)
$$

uniformly in $A_{0}$, and from this the conclusion of Corollary 2 follows at once.

(b) Suppose $\lim _{N \rightarrow \infty} \prod_{i=1}^{N} P\left(A_{i}\right)>0$. Then $\sum_{i=1}^{\infty} P\left(A_{i}^{c}\right)<\infty$. Now choose $N$ so large that $\prod_{i=1}^{N} P\left(A_{i}\right)-\prod_{i=1}^{\infty} P\left(A_{i}\right)<\epsilon / 2$ and such that $\sum_{i=N}^{\infty} P\left(A_{i}^{c}\right)<\epsilon / 2$. Then it is easily verified that

$$
P\left(A_{0} T^{i_{1}} A_{1} \cdots T^{i_{N}} A_{N}\right)-P\left(A_{0} T^{i_{1}} A_{1} \cdots T^{i_{h}} A_{k}\right)<\epsilon / 2
$$

uniformly in $A_{0}$ and for $k>N$. The proof is concluded by applying Corollary 1 once more.

\section{REFERENCE}

1. J. R. Blum and D. L. Hanson, On invariant probability measures. II, Pacific J. Math. 11 (1961), 63-71.

University of New Mexico and Sandia Corporation 